\title{
Extinction does not depend upon degradation of event memories
}

\author{
WESLEY J. KASPROW, TODD R. SCHACHTMAN, HAYDEE CACHEIRO, \\ and RALPH R. MILLER \\ State University of New York, Binghamton, New York
}

\begin{abstract}
Rescorla's "event-memory" hypothesis posits that extinction decrement is due in part to a weakening of a central unconditioned stimulus (US) representation that is shared by all stimuli associated with a particular US. Thus, extinction of one stimulus conditioned to a US should decrease conditioned responding to a second stimulus that has been independently conditioned to the same US. In each of the present experiments, after excitatory conditioning to two stimuli separately, some rats experienced repeated nonreinforced presentations of one of the conditioned stimuli, while others were given no such exposures. Despite parametric variations across experiments, no reliable attenuation of suppression to the nonextinguished stimulus was evident during testing. Tests for suppression to the extinguished stimulus demonstrated a traditional extinction decrement.
\end{abstract}

Echoing Konorski (1948), Rescorla (1973, 1974) argued that the performance of a first-order conditioned response (CR) depends not only upon an association between the conditioned stimulus (CS) and unconditioned stimulus (US), but also upon the current state of a memorial "US representation." Consistent with this view, postacquisition procedures that directly devalue the hypothesized US representation established during conditioning are reported to be effective in reducing the CR to first-order CSs, for example, the habituation of an aversive loud-noise US (Rescorla, 1973). Similarly, procedures designed to enhance the US representation after conditioning are seen to increase the CR to first-order CSs, for example, the postacquisition presentation of a more intense US in the absence of the CS (Rescorla, 1974).

According to the "event-memory" view of conditioning, modification of the US representation may contribute to the CR decrement that arises during extinction of a CS. Within this framework, the association between CS and US is not necessarily weakened by nonreinforced presentations of the CS; rather, the subject's memorial representation of the US becomes degraded and loses its ability to evoke a CR. In other words, following extinction, the subject may still retain the CS-US association, but the US trace reactivated by the CS on a postextinction test trial has been reduced to lower affective value than the US trace at the end of conditioning. Because the memorial US representation is assumed by the event-memory hypothesis to be common to all CSs conditioned to a US, degradation of the US memory by extinction of

This research was supported by NIMH Grant 33881, NIH Biomedical Research Support Grant SO7RR07149, and SUNY University Research Award 7479. Reprint requests should be addressed to Ralph R. Miller, Department of Psychology, State University of New York, Binghamton, New York 13901. one CS should diminish conditioned responding to other CSs that are associated to that US.

The present research was designed to test this prediction in a straightforward manner. Using rats in lick suppression (Experiment 1) and barpress suppression (Experiments 2 and 3) preparations, we separately paired two CSs with a common US. Then for some subjects, one CS was extinguished, while other animals experienced no extinction. Finally, a test for conditioned suppression to the nonextinguished CS was conducted. A reduction in the $C R$ by the extinguished animals on this test would support the notion that extinction of one CS weakens a US representation that is common to all CSs associated with this US, whereas a failure to find any such effect would support the traditional associationist view that suggests that extinction is CS specific. To confirm the sensitivity of the present preparation to extinction effects, the CR to the extinguished CS was also evaluated.

\section{EXPERIMENT 1}

\section{Method}

Subjects and Apparatus. Thirty-six naive male Sprague-Dawleydescended rats (Holtzman, Inc., Madison, WI) weighing 173-300 g were used. The subjects were housed individually in wire-mesh cages. A week before starting the study, the animals were gradually deprived of water and finally stabilized on 10-min/day access. Purina Lab Chow was available ad lib in the home cage. The apparatus, 12 Plexiglas chambers with grid floors, has in detail previously been described (Kasprow, Cacheiro, Balaz, \& Miller, 1982).

Procedure. On Days 1-3, all subjects were given 30-min sessions of lick training in the chambers. As an index of adaptation to the apparatus, the latencies to complete the first 25 and the second 25 licks were recorded during these sessions. There were no appreciable differences among animals on either latency measure on Day 3; therefore, nine subjects were assigned randomly to each of four treatment conditions: $N / T, N / L, T-/ L$, and $L-/ T$.

On-baseline Pavlovian conditioning was conducted during 1-h sessions on Days 4-6. Four trials per day, the first occurring $4.5 \mathrm{~min}$ into the session, were distributed pseudorandomly with an 
average intertrial interval of $13.8 \mathrm{~min}$. In each session, all subjects received two pairings of a 10-sec flashing light (L: .5 sec on/.5 sec off, 7.5 W) with a 2-sec, 1.2-mA footshock and two pairings of a 10-sec tone (T: $1300 \mathrm{~Hz}, 5 \mathrm{dBC}$ above background) with an identical footshock. Shock onset was concurrent with CS offset. The sequence of pairings (LTLT or TLTL) was counterbalanced across subjects on each day; additionally, for each subject, the Day 5 sequence was the opposite of that received on Days 4 and 6.

On Day 6, the session length was extended to $2.5 \mathrm{~h}$, and Groups $\mathrm{T}-/ \mathrm{L}$ and $\mathrm{L}-/ \mathrm{T}$ began their respective extinction treatments following the last conditioning trial. Group $T-/ \mathrm{L}$ received 14 nonreinforced presentations of the tone, and Group $L-/ T$ received 14 nonreinforced flashing-light presentations. The CS-only presentations were distributed pseudorandomly over the 1.5 -h extinction phase of the session, with the first presentation occurring 76.5 min after the rat had been placed in the chamber. Groups N/T and $N / L$ received no extinction during this time, but remained in their chambers. Extinction treatment continued on Days 7-9. Session length on these days was $1.5 \mathrm{~h}$, and the treatment was identical to that in the last $1.5 \mathrm{~h}$ of Day 6. Thus, across days, Group $\mathrm{L}-/ \mathrm{T}$ received a total of 56 nonreinforced light presentations and Group $\mathrm{T}-/ \mathrm{L}$ received an identical number of nonreinforced tone exposures. In addition to providing nominal treatment, the 1.5-h sessions on Days 6-9 served as recovery sessions that extinguished any context-US associations and restored animals to their Day 3 lick baselines. Latency to complete the first 25 and the second 25 licks after placement in the chamber was recorded daily for each subject.

On Day 10, a test of conditioned suppression was conducted. Each rat was returned to its conditioning chamber and was allowed to complete 25 licks. Upon emission of Lick 25, the test CS was initiated and remained on until Lick 50 had been completed. Use of this procedure ensured that each rat was licking at the time of CS presentation. Groups N/T and $\mathrm{L}-/ \mathrm{T}$ were tested for suppression to the tone, and Groups N/L and T-/L were tested for suppression to the flashing light. For each animal, both the latency to complete the first 25 licks in the absence of the test stimulus and the latency to complete the second 25 licks in the presence of the test stimulus were recorded. A ceiling value of $3,600 \mathrm{msec}$ was used during the test. On Day 11, a similar test was performed with all animals, but the nontarget CS (i.e., the one not used on Day 10) was presented.

\section{Results and Discussion}

One subject in Group L - /T and another in Group $\mathrm{N} / \mathrm{T}$ failed to lick after conditioning; therefore, these two animals were eliminated from the study. The latencies to Lick 25 and from Lick 25 to Lick 50, recorded from Day 3. Days 7-9 and Days 10-11 for the remaining 34 subjects, were transformed to $\log$ latencies to permit the use of parametric statistics. On none of these measures did any of the animals reach the 3,600-sec ceiling. Analyses of pretest log latencies to Lick 25 yielded no between-group differences (all ps >.10). The mean log latencies ( \pm SEs) for the second 25 licks on Day 10 were $1.74( \pm .10)$ sec for Group $\mathrm{N} / \mathrm{T}, 1.90( \pm .12) \mathrm{sec}$ for Group L-/T, $1.98( \pm .11)$ sec for Group N/L, and $1.67( \pm .15)$ sec for Group $T-/ L$. A treatment (extinction vs. no extinction) $x$ test stimulus (tone vs. light) analysis of variance indicated no effect of treatment or of test stimulus (ps > .25). The treatment $x$ test stimulus interaction also failed to achieve significance $[F(1,30)=3.79, p>.05]$.

On Day 11, the mean $\log$ test latencies ( \pm SEs) were $1.90( \pm .09) \mathrm{sec}$ for Group N/T, $1.29( \pm .16) \mathrm{sec}$ for Group L $-/ \mathrm{T}, 1.94( \pm .15)$ sec for Group N/L, and $1.40( \pm .08) \mathrm{sec}$ for Group $\mathrm{T}-/ \mathrm{L}$. The analysis yielded a main effect for treatment $[\mathrm{F}(1,30)=20.33$, $\mathrm{p}<.001]$. The main effect of test stimulus and the treatment $x$ test stimulus interaction were not significant (ps $>.25$ ). A test for carry-over effects of Day 10 testing to the Day 11 test was conducted by comparing groups in the no-extinction condition that were tested on the same stimulus. These comparisons found that the performance of Group N/T on Day 10 was comparable to that of Group N/L on Day 11 $[t(15)=1.07, p>.20]$ and that the performance of Group N/L on Day 10 did not differ from Group N/T on Day $11[t(15)=0.58, p>.20]$.

The present results provide a test of the view that extinction decrement results in part from a degraded US representation. According to this theory, extinction of one stimulus, by devaluing a common US representation, should reduce the CR to another stimulus that was similarly conditioned to that US but that was not extinguished. The data fail to support that prediction. Although our extinction procedure produced substantial loss of the CR to the extinguished CS, no such decrement was evidenced when the nonextinguished stimulus was tested.

The reason for the tendency toward a treatment $x$ test stimulus interaction on Day 10 is not readily apparent. It is unreasonable to conclude that extinction to the tone modestly weakened the US representation but that extinction to the light enhanced it. Perhaps it is most prudent to attribute the tendency to chance variation in the data, as is suggested by the statistical analysis. However, we decided to conduct further experiments that might provide an unequivocal test of cross-stimulus extinction effects.

\section{EXPERIMENT 2}

Using procedures designed to minimize the difficulties encountered in Experiment 1, Experiment 2 investigated the effect of extinction of one CS upon a second CS. Because the extinction of differentmodality CSs (tone and light) had produced slightly asymmetrical effects upon suppression to the nonextinguished CS in Experiment 1, Experiment 2 attempted to avoid this problem by using two auditory CSs (tone and clicks). Additionally, barpress suppression, rather than lick suppression, was employed because conditioned barpress suppression is more sensitive to low levels of fear than is lick suppression. This allowed the use of a less intense footshock, which minimized direct context-US associations confounding our measure of fear.

\section{Method}

Subjects and Apparatus. Twelve male and 12 female naive albino rats bred in the laboratory from Holtzman stock served as the subjects. The males weighed $180-310 \mathrm{~g}$; the females weighed 150-225 g. The subjects were maintained as in Experiment 1 . The apparatus, 12 standard operant chambers with grid floors, has been previously described in detail (Kasprow, Schachtman, \& Miller, in press). 
Procedure. All subjects were initially shaped to barpress for water reinforcement during 60 -min sessions. Gradually, a variableinterval 60-sec (VI-60) schedule was instituted. The subjects were allowed five sessions of barpressing on VI-60 before any stimuli were presented. On the day before the start of conditioning, the tone (T: $3000 \mathrm{~Hz}, 20 \mathrm{dBC}$ above background) and the clicks (C: $25 / \mathrm{sec}, 14 \mathrm{dBC}$ above background) were each presented once for 60 $\mathrm{sec}$ to all subjects to attenuate any conditioned reactions to them.

Three days of on-baseline Pavlovian conditioning began on the next day. Four trials per day were administered $(10,23,40$, and 55 min into the 60-min session). In each session, all subjects received two pairings of a $60-\mathrm{sec}$ tone with footshock $(.5 \mathrm{~mA}$, $250 \mathrm{msec}$ ) and two pairings of a 60 -sec click train with the same footshock. The sequence of pairings (TCTC or CTCT) was counterbalanced across subjects on each day; the order of trials was reversed on the second conditioning day. Assignment to conditioning sequences was counterbalanced for sex and baseline barpressing rates. Acquisition of conditioned suppression was monitored by forming $A /(A+B)$ suppression ratios; $A$ represents the number of barpresses during the $60-\mathrm{sec} C S$ period, and $B$ represents responding during the $60 \mathrm{sec}$ immediately preceding CS onset.

Upon completion of conditioning, six rats were assigned to each of four groups (N/T, N/C, C - /T, and T - /C; counterbalanced for sex and order of pairings), and the extinction phase of the experiment began. During each of four 1-h extinction sessions, Group $\mathrm{C}-/ \mathrm{T}$ received four nonreinforced click exposures, and Group $\mathrm{T}-/ \mathrm{C}$ received the same number of nonreinforced tone presentations. These exposures were scheduled for 10, 23, 40, and $55 \mathrm{~min}$ into the session. Groups N/T and N/C barpressed in the absence of CS presentations during this time.

Tests for conditioned suppression were carried out on the day following completion of extinction. Groups N/T and C-/T received four nonreinforced tone presentations, thus providing measures of unimpaired acquisition and potential cross-stimulus extinction effects to the tone, respectively. Groups N/C and T-/C received four nonreinforced click presentations, thereby providing analogous information concerning the clicks.

\section{Results and Discussion}

The data of two subjects from each of Groups $\mathrm{C}-/ \mathrm{T}$ and N/T and of one subject from Group $\mathrm{T}-/ \mathrm{C}$ were lost due to equipment failure. Suppression data from the remaining subjects for the first trial of each conditioning and extinction day and from each test trial are plotted in Figure 1. Conditioned suppression developed quickly and similarly across days to both CSs. Separate analyses of variance (ANOVAs) on the firsttrial scores during conditioning showed a reliable increase over days in suppression to the clicks $[F(2,34)=$

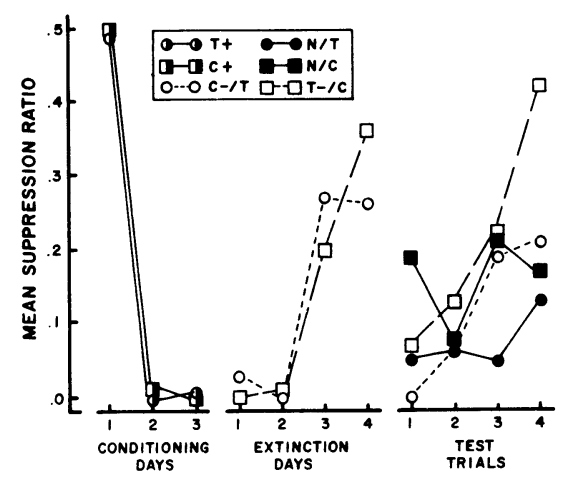

Figure 1. Mean suppression ratios for each group on the first trial of each conditioning and extinction day and for each test trial with the nonextinguished CS.
$126.87, p<.001]$ and the tone $[F(2,34)=175.49$, $\mathrm{p}<.001]$. During the extinction phase of the experiment, loss of suppression proceeded similarly for the clicks in Group $\mathrm{C}-/ \mathrm{T}$ and the tone in Group $\mathrm{T}-/ \mathrm{C}$. A stimulus (tone vs. clicks) $x$ days ANOVA on the first-trial scores confirmed this. The days main effect was significant $[F(3,24)=12.85, p<.001]$, but the stimulus main effect $[F(1,8)<1]$ and the stimulus $x$ days interaction $[F(3,24)<1]$ were not.

Analysis of the test-trial suppression ratios revealed no effect of extinction to one CS upon the CR to the nonextinguished CS. A treatment (no extinction vs. extinction) $\mathrm{x}$ test stimulus (tone vs. clicks) ANOVA on the first test trial found no main effect of treatment $[F(1,13)=1.33, p>.20]$ or of test stimulus $[F(1,13)=2.10, p>.10]$ and no interaction of these variables $[F(1,13)<1]$. The groups were similarly nondifferentiated on Trials 2,3 , and $4[\mathrm{Fs}(1,13)<$ 2.69 , ps $>.10$ ]. Inspection of individual subject scores on Test Trial 4 showed that the lack of suppression in Group $\mathrm{T}-/ \mathrm{C}$ was due primarily to one animal (suppression ratio $=.75$ ).

These results provide another demonstration of the lack of cross-stimulus extinction, despite the effectiveness of nonreinforced exposures in producing extinction. Moreover, these results were obtained without the disturbing tendency toward the interaction between treatment and stimulus that was seen in Experiment 1.

Although Experiment 2 indicated a lack of crossstimulus extinction effects, the suppression produced by the conditioning trials was perhaps too substantial to allow for observation of the degradation of the US representation. That is, the intact US representation in the no-extinction groups may have been stronger than that in the extinction groups, but all groups may still have been below a behavioral threshold for seeing these differences. Supporting this possibility is the fact that at least half the subjects in each group had zero suppression-ratio values on Test Trial 1. Taking this position would force one to conclude that the manifest loss of suppression to the CSs during the extinction phase of the experiment was due primarily to weakening of the CS-US association rather than to US representation devaluation. But the event-memory hypothesis does not exclude the possibility that extinction decrement occurs in part from the loss of the CS-US association; it simply argues that there are additional causes.

\section{EXPERIMENT 3}

Experiment 3 sought to establish a weaker US representation than the one that existed in Experiment 2, thereby making any extinction-induced changes in it more readily manifest. This was accomplished by using a less intense footshock US. To reduce further the likelihood of context-US associations, the session length was extended to $2 \mathrm{~h}$, allowing for longer intertrial intervals during conditioning. 


\section{Method}

Subjects and Apparatus. Twenty-four naive male albino rats (purchased from Holtzman, Inc.) weighing 160-235 g served as the subjects. The rats were maintained as in the previous experiments. The apparatus was that of Experiment 2.

Procedure. Initial barpress training and preconditioning exposure to the CSs were conducted as in Experiment 2, except session length was $2 \mathrm{~h}$. Pavlovian conditioning, extinction, and testing were conducted as in Experiment 2, with the following exceptions. Footshock during conditioning was reduced to $.4 \mathrm{~mA}$ and $200 \mathrm{msec}$, session length was $2 \mathrm{~h}$, and all stimuli were scheduled 10, 45, 70, and $100 \mathrm{~min}$ into the session.

\section{Results and Discussion}

Suppression scores from the first trial of each conditioning and extinction day and from each test trial are depicted in Figure 2. Conditioned suppression was established rapidly to each CS, as confirmed by separate days-effect ANOVAs on the first-trial scores on the clicks $[\mathrm{F}(2,46)=109.26, \mathrm{p}<.001]$ and the tone $[F(2,46)=107.73, p<.001]$. Groups $C-/ T$ and $\mathrm{T}-/ \mathrm{C}$ extinguished similarly to the clicks and tone, respectively, as indicated by a stimulus $\mathrm{x}$ days ANOVA on the first-trial scores of the extinction days: The days main effect was significant $[F(3,30)=21.81$, $\mathrm{p}<.001]$, whereas the stimulus main effect $[\mathrm{F}(1,10)=$ $1.05, \mathrm{p}>.20$ ] and the stimulus $x$ days interaction $[F(3,30)<1]$ were not.

Separate stimulus $x$ treatment ANOVAs were carried out on the data from each test trial. The main effect of treatment was nonsignificant on every trial $[F s(1,20)<1]$, as were the main effect of stimulus $[\mathrm{Fs}(1,20)<2.07$, ps $>.10]$ and the interaction of these factors $[\mathrm{Fs}(1,20)<3.00$, ps $>.10]$.

The results of this experiment demonstrate the null effects of extinction of the "other" CS upon the manifest CR to the target CS in a situation in which the US representation was substantially weaker than that of Experiment 2. The effect of footshock reduction was most pronounced during extinction. Loss of suppression proceeded more rapidly and was more complete in Experiment 3 than in Experiment 2. Yet, no consistent effect of extinction of one CS was observed when the nonextinguished CS was tested.

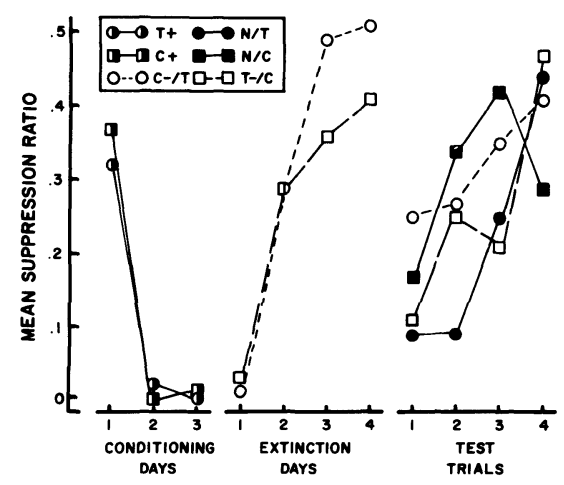

Figure 2. Mean suppression ratios for each group on the first trial of each conditioning and extinction day and for each trial during testing with the nonextinguished CS.

\section{GENERAL DISCUSSION}

The present series of experiments failed to find a reliable effect of extinction of one CS upon the CR elicited by a second nonextinguished CS despite the use of various CS, US, and baseline response parameters. In contrast, "traditional" extinction decrements were quite pronounced in each of these three studies. The nonsignificant tendencies toward cross-stimulus effects that were evident in Experiments 1 and 3 are left unexplained, but they do not fit the framework of the event-memory hypothesis. For example, the nonsignificant tendencies toward cross-stimulus effects, when they did occur, were confined to only one stimulus condition. Because the US representation is alleged to be common to all CSs conditioned to the US, any asymmetry in cross-stimulus extinction must lie outside the scope of the event-memory hypothesis. A potential explanation of the asymmetry in the present experiments is that presenting the different CSs in the context during extinction had differential effects upon the rate of extinction of the context itself, thus altering its contribution to manifest suppression (see Wagner, 1969). However, such an explanation does not undermine the main thrust of the present experiments: Extinction decrement is CS specific, and therefore is not amenable to explanation by hypothesized modification of a general US representation.

Perhaps the most important difference between the present failure to find cross-stimulus extinction effects and the positive effects obtained by Rescorla and Cunningham (1977) using the erasure paradigm is the nature of the procedure used to produce the "intact" US representation that was to be modified. The present study relied on the original conditioning trials to establish a strong US representation, whereas Rescorla and Cunningham (1977) "reactivated" the US representation after initial extinction of the target CS. The discrepancy in results between the two sets of studies suggests that the US representation produced by conditioning alone and the US representation(s) produced by conditioning plus the reinstatement procedure may be fundamentally different. Specifically, the results suggest the possibility that the US representation after US reinstatement is more susceptible to further modification than is the US representation arising from original conditioning. Richards and Sargent (1983) recently reported results from an independently conducted series of experiments that are similar to the present findings and likewise concluded that US reinstatement may be necessary for seeing cross-stimulus extinction effects.

\section{REFERENCES}

Kasprow, W. J., Cacheiro, H., Balaz, M. A., Miller, R. R. (1982). Reminder-induced recovery of associations to an overshadowed stimulus. Learning and Motivation, 13, 155-166.

Kasprow, W. J., Schachtman, T. R., \& Miller, R. R. (in press). Associability of a previously-conditioned stimulus as a function of qualitative changes in the US. Quarterly Journal of Experimental Psychology.

KonORSKI, J. (1948). Conditioned reflexes and neuron organization. Cambridge: Cambridge University Press.

REscorla, R. A. (1973). Effect of US habituation following conditioning. Journal of Comparative and Physiological Psychology, 82, 137-143.

RESCORLA, R. A. (1974). Effect of inflation of the unconditioned stimulus value following conditioning. Journal of Comparative and Physiological Psychology, 86, 101-106.

Rescorla, R. A., \& Cunningham, C. L. (1977). The erasure of reinstated fear. Animal Learning \& Behavior, 5, 386-394.

Richards, R. W., \& Sargent, D. M. (1983). The order of presentation of conditioned stimuli during extinction. Animal Learning \& Behavior, 11, 229-236.

WAGNER, A. R. (1969). Stimulus selection and a "modified continuity theory." In G. H. Bower \& J. T. Spence (Eds.), The psychology of learning and motivation (Vol. 3, pp. 1-43). New York: Academic Press.

(Revision received for publication January 21, 1984.) 